\title{
BMJ open The effect of a web-based depression intervention on suicide ideation: secondary outcome from a randomised controlled trial in a helpline
}

\author{
Helen Christensen, ${ }^{1}$ Louise Farrer, ${ }^{2}$ Philip J Batterham, ${ }^{2}$ Andrew Mackinnon, ${ }^{3}$ \\ Kathleen M Griffiths, ${ }^{2}$ Tara Donker ${ }^{1}$
}

To cite: Christensen $\mathrm{H}$, Farrer L, Batterham PJ, et al. The effect of a web-based depression intervention on suicide ideation: secondary outcome from a randomised controlled trial in a helpline. BMJ Open 2013;3:e002886. doi:10.1136/bmjopen-2013002886

- Prepublication history and additional material for this paper is available online. To view these files please visit the journal online (http://dx.doi.org/10.1136/ bmjopen-2013-002886).

\section{- Additional material is published online only. To view please visit the journal online (http://dx.doi.org/10. 1136/bmjopen-2013- 002886).}

Received 15 March 2013 Revised 26 April 2013 Accepted 20 May 2013

This final article is available for use under the terms of the Creative Commons Attribution Non-Commercial 3.0 Licence; see http://bmjopen.bmj.com

For numbered affiliations see end of article.

\section{Correspondence to}

Professor Helen Christensen; h.christensen@unsw.edu.au

\section{ABSTRACT}

Objectives: The effect of web-based interventions for depression on suicide ideation in callers to helplines is not known. The aim of this study was to determine if web-based Cognitive Behaviour Therapy (CBT) with and without telephone support is effective in reducing suicide ideation in callers to a helpline compared with treatment as usual (TAU). A secondary aim was to examine the factors that predict change in suicide ideation. Putative predictors included level of baseline depression, suicide behaviour, baseline anxiety and type of intervention.

Design: Randomised controlled trial.

Setting: Lifeline, Australia's $24 \mathrm{~h}$ telephone counselling service participants: 155 callers to a national helpline service with moderate-to-high psychological distress.

Interventions: Participants were recruited and randomised to receive either 6 weeks of internet CBT plus weekly telephone follow-up; internet CBT only; weekly telephone follow-up only or a wait-list TAU control group.

\section{Primary and secondary outcome measures:}

Suicidal ideation was measured using four items from the 28-item General Health Questionnaire. Predictors of change in ideation were tested using logistic regression analysis.

Results: Regardless of the intervention condition, participants showed significant reductions in suicidal ideation over 12 months $(p<0.001)$. Higher baseline suicidal behaviour decreased the odds of remission of suicidal ideation at postintervention (OR 0.409, $\mathrm{p}<0.001$ ). However, change in depression over the course of the interventions was associated with improvement in suicide ideation (OR 1.165, $\mathrm{p}<0.001)$.

Conclusions: Suicide ideation declines with and without proactive intervention. Improvements in depression are associated with the resolution of suicide ideation. Specific interventions focusing on suicide ideation should be further investigated.

Trial registration: Controlled-Trials.com ISRCTN93903959.

\section{INTRODUCTION}

Suicide prevention interventions are delivered every day through helplines and

\section{ARTICLE SUMMARY}

Article focus

- To evaluate the effectiveness of a web-based Cognitive Behaviour Therapy (CBT) intervention on suicide ideation in a helpline.

- To examine predictors of suicide ideation resolution.

Key messages

- Irrespective of the intervention condition, participants' suicidal ideation declined over time at post-test and at 6 months. Declines in suicidal ideation remained significant at 12-month follow-up.

- Suicide ideation reduces significantly more in those individuals who also had resolving depression.

- While CBT internet interventions are significantly more effective in resolving depression symptoms compared with control conditions, these operate no more successfully than current call centre practices in resolving suicide ideation.

- Our findings demonstrate the importance of not excluding suicidal participants from interventions designed to target depression.

Strengths and limitations of this study

- The study used a randomised controlled trial.

- The addition of the treatment as usual control condition is a significant improvement over previous trials.

- As all scores were initially high at baseline, the lower scores on the second occasion of measurement may have been due to reversion to the mean.

through chat in call centres. ${ }^{1}$ In Australia, Lifeline reports that over 50 high-risk telephone calls are answered daily. ${ }^{2}$ However, despite the load taken by helplines in responding to callers at risk, there has been little investigation of the role of these services in reducing suicide risk, or in reducing risk factors associated with suicide risk, such as depression. 
Until now, the research evidence from suicide helplines has largely focused on evaluating interventions using the telephone, and to our knowledge, only one randomised controlled trial (RCT) has been conducted. This study ${ }^{3}$ evaluated the effects of two brief telephone psychotherapies in reducing general distress (Solution Focussed Brief Therapy (SFBT) and Common Factors Therapy (CFT) ) compared with a waitlist control condition using 85 individuals recruited via a suicide hotline. Outcome measures included the Brief Psychiatric Rating Scale and the Beck Depression Inventory. Dropout was high in the SFBT condition (50\%) compared with the CFT condition (37\%), and across both conditions, dropout occurred before the first session. Relative to the waitlist control, significant reductions in several measures of depression and anxiety symptoms were found in participants who completed the interventions, with effect sizes estimated in the range of 0.67 and 0.93 . However, outcomes on suicide-specific measures were not reported. A highly cited, although uncontrolled, study used a follow-up design (pre-post outcomes) to evaluate the effectiveness of a helpline for non-suicidal crisis callers $(n=1617$, of whom 801 provided follow-up data). ${ }^{4}$ Callers' levels of crisis and hopelessness were rated during the calls and found to decrease during the course of the call. In a second study, focusing on 1085 participants who were suicidal, Gould et a $\bar{l}$ found that levels of intent to die over the course of a call decreased and that there were continuing falls in hopelessness and psychological pain over the continuing weeks.

Web-based interventions are now more frequently used in helpline services, and research of their usefulness is emerging in community settings. Two studies of online e-health applications targeting either risk factors for suicide (depression) or suicide ideation were published in 2012. The first was an uncontrolled service evaluation which found a $30 \%$ reduction in suicide ideas $^{6}$ over the course of a depression web program. The second was an RCT of an online intervention which examined the effectiveness and cost effectiveness of an automated six module, 6 week intervention specifically targeting suicide ideation for individuals with mild-to-moderate suicidal thoughts. The intervention was based on Cognitive Behaviour Therapy (CBT), with Dialectical Behaviour Therapy, Problem Solving Therapy and Mindfulness-Based Cognitive Therapy included. Significant reductions in suicidal thoughts were found compared to the control condition. The mean incremental cost-effectiveness ratio was estimated to be $€ 34727$ (US\$41 325) for an additional treatment response, indicating annual cost savings per treatment responder. $^{7}$

In 2012, we published the results of an RCT investigating the effects of a web application in reducing depression and anxiety in a large helpline. ${ }^{8} 9$ This trial compared four conditions: a treatment as usual condition (TAU), where the participant could call the crisis line at any time; a telephone call back service where the participant was contacted for 10 min systematically once a week for 6 weeks; a CBT internet program for depression combined with an evidence-based depression information website and a combined condition which incorporated telephone call back and the web programs. Both the web programs alone, and the web programs in combination with the telephone call back, were effective at 12 months in reducing depression symptoms, relative to TAU. This study also measured suicide ideation, and in this paper we report the results using this outcome measure.

Suicide ideation is a substantial risk factor for suicide attempt. Theories of suicide causation point to the importance of depression and hopelessness in leading to increased levels of suicide ideation and attempts. ${ }^{10}$ We hypothesised that suicide ideation would be reduced over time in those conditions which successfully reduced depression. Accordingly, we first examined the effect of the four interventions conditions on suicide ideation. For suicide ideation, we hypothesised that the web CBT intervention focusing on reducing depression-with or without the telephone callback-would result in a significantly greater reduction of suicide ideation than the other two conditions, which did not include interventions specifically aiming to reduce depression. We then used logistic regression to explore which factors, in addition to any effect of the intervention, predicted changes in suicide ideation at post-test. These factors were baseline suicidality, baseline depression, baseline anxiety, age, gender, education, workforce participation and changes in depression and anxiety over the course of the intervention (pretest to post-test).

\section{METHOD}

Data for this study were collected as part of an RCT of the impact of a web-based CBT and psychoeducation intervention on depression symptoms. ${ }^{9}$ The trial comprised four arms: (1) a web-based CBT intervention; (2) a web-based CBT intervention delivered in conjunction with a telephone call back; (3) the proactive call back telephone line alone and (4) TAU, which consisted of an opportunity for the participant to contact the helpline if needed.

\section{Participants}

Participants were 155 callers to Lifeline, Australia's $24 \mathrm{~h}$ telephone counselling service, recruited between July 2007 and January 2009. High distress or acutely suicidal participants were excluded. A total of 910 callers agreed to be screened by telephone. Of these, $142(15.6 \%)$ were subsequently unable to be contacted, $61(7 \%)$ were later unwilling to participate and 337 (37\%) did not meet the eligibility criteria. Respondents were not eligible for inclusion in the trial if they (1) scored less than 22 on the Kessler Psychological Distress Scale ${ }^{11}$ (138/ $337,41 \%)$, a brief screening scale used clinically and epidemiologically, (2) had, by self-report, a diagnosis 
of schizophrenia or bipolar disorder $(89,26 \%)$ or (3) did not have internet access $(67,20 \%)$. Ineligible participants were offered brochures sent by mail containing information about the web intervention used in the trial. Of the 370 people eligible for inclusion in the trial, 155 completed informed consent procedures and preintervention assessments, and were randomly assigned to the trial conditions. The trial flow is illustrated in figure 1.

\section{Procedure}

Following screening and informed consent procedures, preintervention data were obtained through a self-report questionnaire mailed to participants. A block randomisation procedure was used, with stratification based on site of recruitment and severity of psychological distress at screening. Allocation of participants to trial conditions was conducted independently by a research assistant not involved in the day-to-day running of the trial. Following randomisation, all participants were contacted by telephone and were mailed the relevant materials for their allocated condition.

\section{Intervention and trial conditions}

The web-only intervention consisted of web-based psychoeducation (in week 1 provided by BluePages: bluepages.anu.edu.au) combined with web-based CBT (in weeks 2-6 provided by MoodGYM: moodgym.anu. edu.au). BluePages is an educational site that provides information on evidence-based interventions for depression. MoodGYM is a first-generation, interactive web application which delivers CBT through a series of five modules, captures user behaviour and self-report symptoms on validated scales and offers an online CBT workbook. Both these web programs have been shown to reduce depression symptoms in community users. ${ }^{12}$ A printed manual containing week-by-week instructions for accessing the web programs (via a login) was mailed to participants at the start of the trial.

In the web with telephone call back condition, participants completed the web intervention and also received a weekly $10 \mathrm{~min}$ telephone call from a Lifeline telephone counsellor. The purpose of these calls was to address any issues associated with the participants' use of the intervention.

In the telephone call back only condition, participants received a weekly 10 min telephone call initiated from a telephone counsellor. These scripted calls focused on various environmental and lifestyle factors associated with depression.

In the TAU condition, participants received neither the call back nor the web interventions, but they were free to use the helpline service as needed. Participants in this condition were wait-listed to receive the web-only intervention following completion of the 6-month follow-up.

As in the TAU condition, participants in all the three other conditions were also able to use the helpline service as needed, which provided usual emergency or support services. Self-reported contacts with the service were taken at each follow-up occasion for each of the four conditions. Any use of this service during the intervention period was additional to the $10 \mathrm{~min}$ telephone calls offered proactively as part of the call back component of the telephone call back condition and the internet plus call back condition.

\section{Measures}

\section{Suicidal ideation}

Suicidal ideation was measured using 4 items from the 28-item General Health Questionnaire (GHQ-28. ${ }^{1}$ The four questions measure suicidal ideation, asking respondents to indicate how they have felt over the last few weeks. The first two items: 'Have you recently felt that life is not worth living' and 'Have you recently found yourself wishing you were dead and away from it all' were scored on the 4-point scale: 'Not at all', 'No more than usual', 'Rather more than usual' or 'Much more than usual'. The remaining two items: 'Have you recently had thoughts of the possibility that you might do away with yourself' and 'Have you recently found that the idea of taking your own life kept coming into your mind' were scored on the 4-point scale: 'Definitely not', 'I don't think so', 'Has crossed my mind' or 'Definitely has'. Responses were then categorised in a binary manner, with the negatively worded response categories (Not at all, No more than usual, definitely not, and I don't think so) scored with a 0 , and the positively worded response categories scored with a 1. Binary scores for each item were summed to provide a total scale score ranging from 0 to 4 , with a higher score indicating higher suicidal ideation. This scoring system is that used for all items and subscores of GHQ-28, which has been validated in Australian populations. ${ }^{13}$ The four suicide items from GHQ-28 have been used extensively by Goldney $e t a l^{14-16}$ to assess suicidal ideation in a number of Australian samples. These items have been validated relative to other wellestablished measures of suicidal intent and hopelessness. ${ }^{17}{ }^{18}$ Cronbach $\alpha$ for the four items at baseline in the present sample was 0.90 .

\section{Predictor variables}

Depression symptoms were measured by the Center for Epidemiologic Studies Depression Scale (CES-D), ${ }^{19}$ a widely used 20-item scale with strong reliability and validity, ${ }^{19} 20$ with scores ranging from 0 to 60 . Anxiety symptoms were assessed using the 14-item anxiety scale from the Depression, Anxiety and Stress Scales (DASS) ${ }^{21}$ Each of these items is rated on a four-point scale from 0 ('did not apply to me at all') to 4 ('applied to me most of the time'), with total scores ranging from 0 to 42 . The reliability and validity of the DASS scale has been demonstrated previously. ${ }^{22} 23$

Demographic variables including age, gender, educational attainment and workforce participation were assessed by self-report in the pretest survey. Educational 


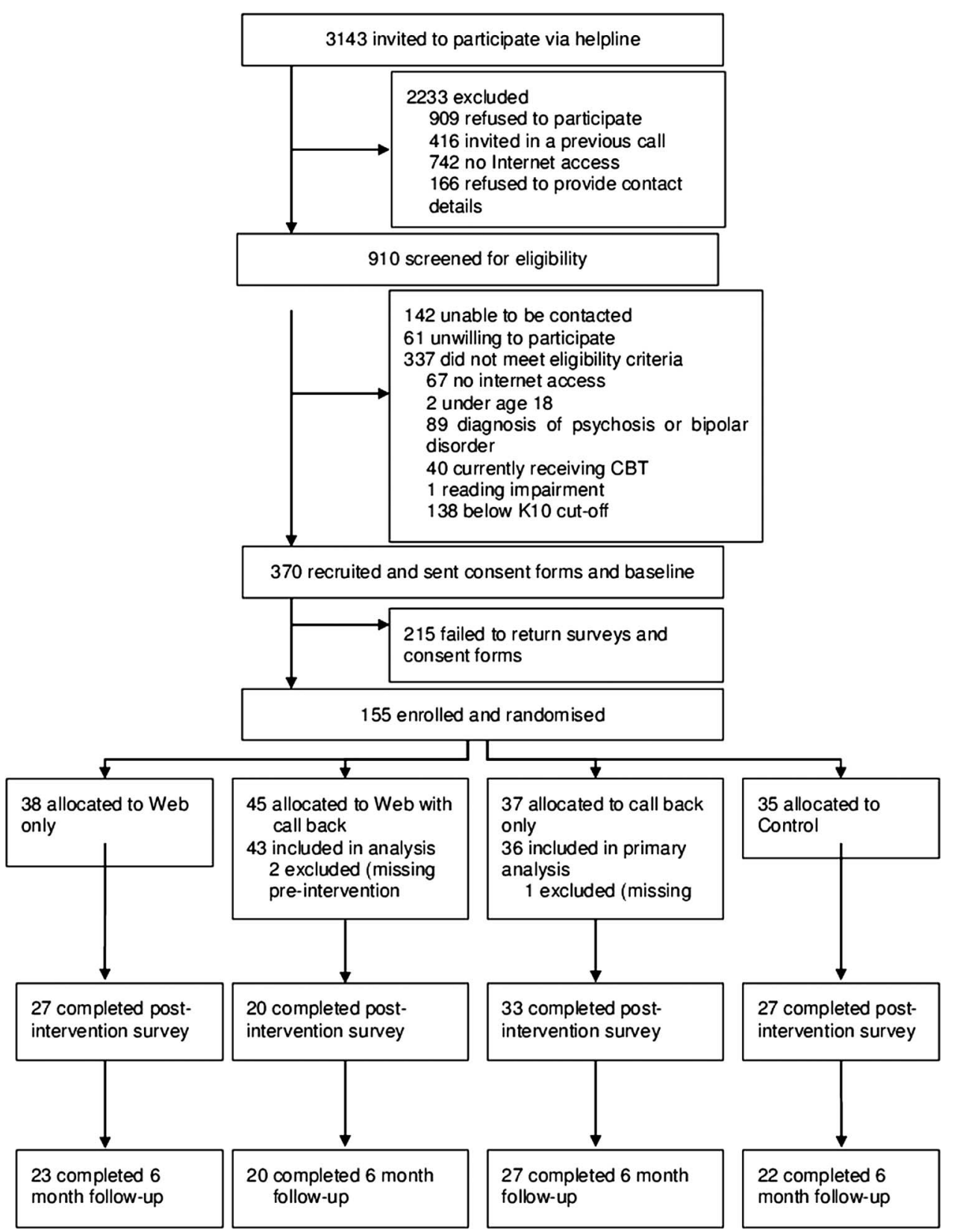

Figure 1 Trial flow diagram.

attainment was based on two items assessing highest level of education, which were used to form an estimate of total number of years of education. Workforce participation was based on an item assessing employment status, with 'unemployed' and 'not in the labour force' categorised as non-participation and employed 'fulltime' or 'part-time' categorised as participation.

\section{Statistical analyses}

Differential changes in suicide ideation across the four conditions were assessed using mixed model repeated measures analysis of variance. Within-person variation was modelled using an unstructured covariance matrix and degrees of freedom were estimated using
Satterthwaite's approximation. This model included the fixed effects of condition, time (pre, post, 6 months, 12 months) and the interaction between condition and time. The presence of a significant interaction between an active intervention and the TAU condition over time would indicate a significant reduction in suicidal ideation for that condition relative to control. Within-condition change in suicidal ideation was also examined using this model. Mixed models yield an intention-to-treat analysis, using all available measurement points for each participant under the assumption that withdrawal data are missing at random. Two participants with missing suicidal ideation scores at baseline were omitted from the analyses. To evaluate whether 
the scoring of the suicidal ideation scale influenced the findings, the models were re-estimated using a continuous score of suicidal ideation, assessed at each time point as the sum of responses to the four ideation items (range 0-12).

Logistic regression was then used to examine predictors of suicidal ideation at postintervention, defined as a suicidal ideation score of 0 . In particular, the hypothesis was assessed that reduction in depression symptoms from preintervention to postintervention predicted remission of ideation. Only completers of the post-test assessment were included in this analysis. A depression symptom change score from preintervention to postintervention was calculated by subtracting postintervention CES-D scores from preintervention scores, with positive scores indicating a reduction/improvement in symptoms. The effects of anxiety symptoms and changes in anxiety were assessed in the same way. Suicidal ideation scores (ranging from 0 to 4 ) were coded categorically as ' 0 ' (score $\geq 1$, indicating some suicidal ideation) or ' 1 ' (score $=0$, no ideation). To control for baseline depression, baseline anxiety, baseline suicidality and intervention condition, these variables were included in the regression simultaneously with the depression change score. In addition, age, gender, education and workforce participation were included in the models due to their potential associations with suicidal ideation.

\section{RESULTS}

\section{Descriptives and changes in suicide ideation}

Table 1 shows the characteristics of the sample at baseline, with mental-health measures for postintervention also presented. The age range was 19-72, with a median age of 42 years. The majority of participants $(57 \%)$ had graduated from high school. Most of the participants were women and approximately half were in the workforce. Depression and anxiety scores were considerably higher than the cut points for the scales used at both time points, although there were decreases from preintervention to postintervention. Likewise, high rates of suicidality were observed, with $62.7 \%$ and $41.5 \%$ reporting suicidal ideation at preintervention and postintervention, respectively.

\section{Mixed models: effect of intervention type on suicidal} ideation

Suicide ideation declined significantly over the 12 -month period $(\mathrm{F}(3,95.4)=15.06, \mathrm{p}<0.001)$. However, differences in change over time between conditions were not significant $(\mathrm{F}(8115.8)=1.13, \mathrm{p}=0.352)$. Figure 2 shows the estimated marginal means for suicidal ideation across measurement occasions.

\section{Within-group contrasts}

As can be seen in table 2, planned contrasts revealed that participants in the call back only and TAU conditions showed significant declines in suicidal ideation from preintervention to postintervention and from preintervention to 6-month follow-up. Declines in suicidal ideation from preintervention to 12-month follow-up were significant for participants in all conditions in which the difference could be assessed.

Table 1 Characteristics of participants

\begin{tabular}{|c|c|c|c|}
\hline & $\mathbf{N}$ & Mean & SD \\
\hline Age & 155 & 41.49 & 12.35 \\
\hline Years of education & 150 & 13.47 & 2.67 \\
\hline CES-D depression score, preintervention & 152 & 36.25 & 10.22 \\
\hline CES-D depression score, postintervention & 107 & 28.68 & 14.40 \\
\hline DASS anxiety score, preintervention & 154 & 13.34 & 8.03 \\
\hline DASS anxiety score, postintervention & 107 & 11.45 & 10.16 \\
\hline GHQ suicidal ideation score-preintervention & 153 & 1.73 & 1.64 \\
\hline \multirow[t]{2}{*}{ GHQ suicidal ideation score-postintervention } & 106 & 1.00 & 1.45 \\
\hline & $\mathrm{N}$ & Count & Per cent \\
\hline Gender & 155 & & \\
\hline Female & & 127 & 81.9 \\
\hline Male & & 28 & 18.1 \\
\hline Workforce participation & 155 & & \\
\hline Participation & & 72 & 46.5 \\
\hline Non-participation & & 83 & 53.5 \\
\hline Treatment condition & 155 & & \\
\hline Internet only & & 38 & 24.5 \\
\hline Internet plus call back & & 45 & 29.0 \\
\hline Telephone call back only & & 37 & 23.9 \\
\hline Treatment as usual & & 35 & 22.6 \\
\hline
\end{tabular}




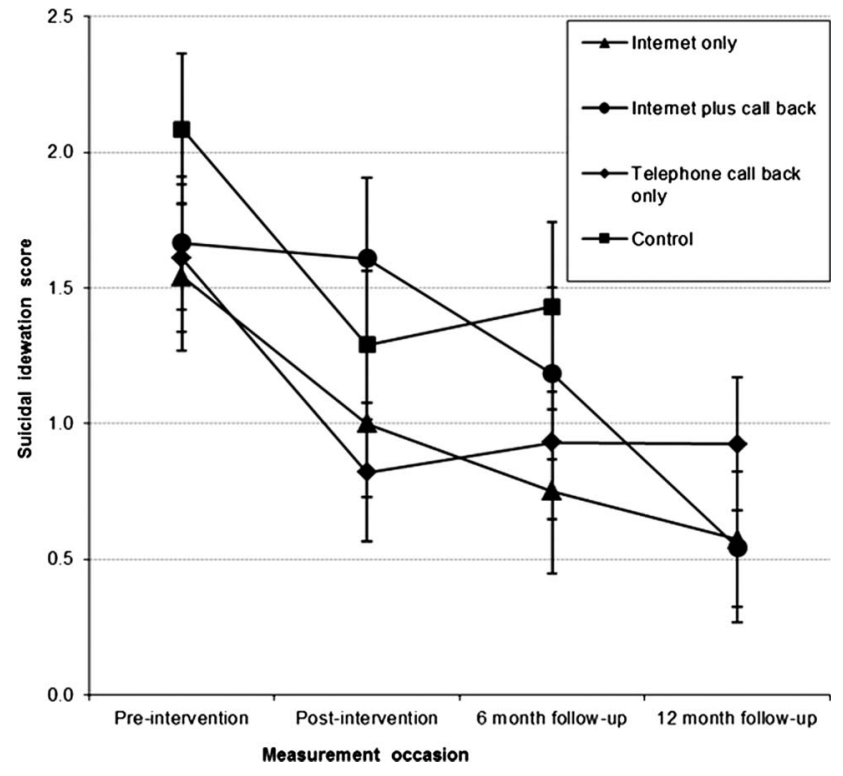

Figure 2 Estimated marginal means for suicidal ideation across measurement occasions.

\section{Between-group contrasts}

No significant differences were found between conditions at postintervention, 6-month follow-up, or 12-month follow-up: internet only vs TAU (post-test: contrast estimate $=0.26 ; \mathrm{p}=0.51 ; 6$-month follow-up: contrast estimate $=$ $-0.14, \mathrm{p}=0.77)$; internet only versus telephone call back only (post-test: contrast estimate $=0.25 ; \mathrm{p}=0.51 ; 6$-month follow-up: contrast estimate $=-0.11, \mathrm{p}=0.80 ; 12$-month follow-up: contrast estimate $=-0.28 ; \mathrm{p}=0.45$ ); internet +telephone call back versus TAU (post-test: contrast estimate $=0.74 ; \quad \mathrm{p}=0.07 ; \quad 6$-month follow-up: contrast estimate $=0.17, \mathrm{p}=0.72)$; internet+telephone call back versus telephone call back only (post-test: contrast estimate $=0.73 ; \mathrm{p}=0.07 ; \quad 6$-month follow-up: contrast estimate $=0.19, \mathrm{p}=0.67 ; 12$-month follow-up: contrast estimate $=-0.44 ; p=0.26)$; telephone call back only versus TAU ( post-test: contrast estimate $=0.01 ; \mathrm{p}=0.99 ; 6$-month follow-up: contrast estimate $=-0.02, \mathrm{p}=0.96)$; internet only versus internet+telephone call back (post-test: contrast estimate $=-0.48 ; \mathrm{p}=0.24 ; 6$-month follow-up: contrast estimate $=-0.31, \mathrm{p}=0.51 ; 12$-month follow-up: contrast estimate $=0.16 ; \mathrm{p}=0.69$ ).

To test whether the lack of between-group effects was related to scaling effects of the suicidal ideation measure, the models were re-estimated using a continuous score for suicidal ideation. As with the original analysis, there was significant reduction over time in suicidal ideation scores $(\mathrm{F}(3,94.5)=14.08, \mathrm{p}<0.001)$. Again, there was no significant interaction between condition and time $(\mathrm{F}(8$, $89.9)=0.81, \mathrm{p}=0.598)$, indicating that there was no significant difference in change of suicidal ideation scores between the intervention groups across the study period.

\section{Effect sizes for suicidal ideation}

At postintervention, effect sizes were 0.10 (95\% CI -0.43 to 0.64$)$ for the internet only condition and $0.04(95 \%$ CI -0.54 to 0.62 ) for the internet plus call back condition, compared with the TAU condition. Compared with call back only, effect sizes were 0.28 (95\% CI -0.24 to 0.79 ) for the internet only condition and 0.41 (95\% CI -0.15 to 0.98 ) for the internet plus call back condition. At 6-month follow-up, effect sizes were 0.36 (95\% CI -0.24 to 0.96 ) for the internet only condition and 0.20 (95\% CI -0.42 to 0.81 ) for the internet plus call back condition, compared with the TAU condition. Compared with telephone call back only, effect sizes were 0.08 (95\% CI -0.48 to 0.63 ) for the internet only condition and 0.07 (95\% CI -0.51 to 0.65 ) for the internet plus telephone call back condition. At 12-month follow-up, effect sizes were 0.28 (95\% CI -0.33 to 0.89 ) for the internet only condition and 0.45 (95\% CI -0.24 to 1.13) for the internet plus call back condition, compared with the call back only condition.

Table 2 Within-group contrast estimates and significance tests for suicidal ideation

\begin{tabular}{|c|c|c|c|c|}
\hline & & \multicolumn{3}{|l|}{ Contrast } \\
\hline & & $\begin{array}{l}\text { Preintervention to } \\
\text { postintervention }\end{array}$ & $\begin{array}{l}\text { Preintervention to } \\
\text { 6-month follow-up }\end{array}$ & $\begin{array}{l}\text { Preintervention to } \\
\text { 12-month follow-up }\end{array}$ \\
\hline Condition & Statistic & & & \\
\hline Internet only & $\begin{array}{l}\text { Contrast estimate } \\
\text { Test value } \\
\text { p Value }\end{array}$ & $\begin{array}{l}-0.54 \\
t(109.8)=-1.98 \\
0.050\end{array}$ & $\begin{array}{l}-0.79 \\
t(101.5)=-2.50 \\
0.016\end{array}$ & $\begin{array}{l}-0.97 \\
t(73.2)=-3.65 \\
<0.001\end{array}$ \\
\hline Internet+telephone call back & $\begin{array}{l}\text { Contrast estimate } \\
\text { Test value } \\
\text { p Value }\end{array}$ & $\begin{array}{l}-0.06 \\
t(121.3)=-0.20 \\
0.85\end{array}$ & $\begin{array}{l}-0.48 \\
t(106.2)=-1.45 \\
0.15\end{array}$ & $\begin{array}{l}-1.12 \\
t(80.8)=-3.91 \\
<0.001\end{array}$ \\
\hline Telephone call back only & $\begin{array}{l}\text { Contrast estimate } \\
\text { Test value } \\
\text { p Value }\end{array}$ & $\begin{array}{l}-0.79 \\
t(105.6)=-3.05 \\
0.003\end{array}$ & $\begin{array}{l}-0.68 \\
t(100.0)=-2.22 \\
0.029\end{array}$ & $\begin{array}{l}-0.68 \\
t(78.0)=-2.61 \\
0.011\end{array}$ \\
\hline Treatment as usual & $\begin{array}{l}\text { Contrast estimate } \\
\text { Test value } \\
\text { p Value }\end{array}$ & $\begin{array}{l}-0.80 \\
t(108.0)=-2.89 \\
0.005\end{array}$ & $\begin{array}{l}-0.66 \\
t(103.1)=-1.96 \\
0.053\end{array}$ & \\
\hline
\end{tabular}




\section{Predictors of suicide ideation}

A logistic regression analysis was designed to measure predictors of suicide ideation change taking account of intervention condition. Consistent with the findings reported above, compared with baseline (M:1.73; SD: 1.64; $\mathrm{N}=153$ ), suicidal ideation decreased significantly in the whole sample at post-test (M:1.00; SD:1.45; N=106), 6-month (M:0.99; SD:1.54; N=91) and 12-month follow-up (M:0.66; SD:1.27; N=56). However, as there was no significant interaction effect between condition and time on suicidal ideation, this interaction was not retained in the model. Table 3 shows the results for the model with interactions excluded. As would be expected, higher levels of suicide ideation at baseline were associated with significantly reduced odds of absence of suicide ideation at post-test. Neither baseline depression nor anxiety was significantly associated with no suicide ideation at post-test. There were also no significant effects of any of the demographic characteristics. However, a greater improvement in depression symptoms from preintervention to postintervention was associated with increased odds of no suicidal ideation at postintervention. At baseline, the majority of participants $(n=93 ; 62 \%)$ reported both depressive symptoms and suicidal ideation. This proportion decreased at all time-points following the interventions (post-test: 44 $(41.5 \%)$; 6-months follow-up: 30 (33\%); 12-months follow-up: $13(23.2 \%))$. Based on McNemar's tests using completers of each assessment, these decreases were significant at all three time points $(p=0.005, p<0.001$ and $\mathrm{p}=0.002$ at post, 6 and 12 months, respectively). There was no significant difference in the proportion of participants with depression but no suicidal ideation, except at 12-month follow-up (baseline: $\mathrm{n}=53$ (35.3\%); post-test: $\mathrm{n}=42 \quad(39.6 \%), \quad 6$-months follow-up: $\mathrm{n}=28 \quad(30.8 \%)$; 12-months follow-up: $18(32.1 \%) ; \mathrm{p}=1.00 ; \mathrm{p}=0.15$ and $\mathrm{p}=0.019$ at post, 6 and 12 months, respectively).

\section{DISCUSSION}

This was the first study to examine the effect of online interventions on suicide ideation in a helpline using an RCT design. In contrast to our hypothesis, results from our study indicated that, irrespective of intervention condition, participants' suicidal ideation declined over time at post-test and at 6 months. Declines were slower to become significant for those randomised to the internet plus call back condition. For all groups, declines in suicidal ideation remained significant at 12-month follow-up. Although there were small effects at post-test and medium effect sizes at follow-up among the intervention conditions, there were no consistent overall effects for conditions. Participants having higher baseline suicide scores were more likely to continue experiencing suicidal ideation at postintervention, while those with greater improvements in depression symptoms were less likely to experience suicide ideation at postintervention.

Several studies have shown reductions of suicide ideation in uncontrolled pre-post trials of counselling or internet CBT. ${ }^{5}{ }^{6}$ This is, to our knowledge, the first RCT of an intervention in a helpline comparing a number of active interventions to TAU. The study is also unique in that it examined predictors of ideation change, taking account of the effect of intervention. As reported previously for this study, ${ }^{9}$ web-based CBT, and web CBT with counsellor call back significantly reduced depression symptoms relative to TAU. These findings indicate that suicide ideation did not follow the same pattern as depression in response to the intervention, and, more generally, that suicide ideation was not strictly tethered to depression. Nevertheless, the regression analysis found that, taking account of intervention type, changes in depression did predict later lower levels of ideation, suggesting that depression change is an important, although not necessarily the only, factor in reducing

Table 3 Logistic regression analyses of remission of suicidal ideations (completers only)*

\begin{tabular}{|c|c|c|c|c|}
\hline & \multirow[b]{2}{*}{ OR } & \multirow[b]{2}{*}{ p Value } & \multicolumn{2}{|l|}{$95 \% \mathrm{Cl}$} \\
\hline & & & Lower & Upper \\
\hline Suicidality score at baseline & 0.409 & $<0.001$ & 0.249 & 0.671 \\
\hline Condition & - & 0.239 & & \\
\hline TAU & 1.000 & - & & \\
\hline Internet only & 1.408 & 0.699 & 0.248 & 8.001 \\
\hline Internet+telephone call back & 0.323 & 0.234 & 0.050 & 2.082 \\
\hline Telephone call back only & 2.442 & 0.284 & 0.476 & 12.512 \\
\hline Baseline depression score & 0.926 & 0.081 & 0.849 & 1.010 \\
\hline Depression change score & 1.165 & $<0.001$ & 1.070 & 1.267 \\
\hline Baseline anxiety score & 1.066 & 0.256 & 0.955 & 1.190 \\
\hline Anxiety change score & 0.986 & 0.836 & 0.859 & 1.130 \\
\hline Gender: F vs M & 0.992 & 0.993 & 0.174 & 5.646 \\
\hline Age & 1.051 & 0.054 & 0.999 & 1.106 \\
\hline Years of education & 0.897 & 0.438 & 0.681 & 1.181 \\
\hline Not in labour force vs Employed & 0.915 & 0.899 & 0.233 & 3.592 \\
\hline
\end{tabular}

*Only main effects included.

TAU, treatment as usual.

Signficant effects are shown in bold. 
ideation. We interpret these findings to suggest that change in depression, in whatever way it is brought about, is a variable contributing to reduction in suicide ideation. A second explanation for the findings is that changes in suicide ideation and changes in depression follow different time trajectories, with ideation resolving faster than depression. The two constructs are likely to be interactive, with ideation reduction assisting in lowering depression symptoms over time. Hopelessness may mediate both depression and ideation but may influence each within different time frames. ${ }^{24}$ Interestingly, a recent meta-analysis ${ }^{25}$ has examined the effects of face-to-face psychotherapy for adult depression on suicidality. It found a non-significant effect of psychotherapy in the reduction of suicidality but a significant effect for depression, consistent with the concept that depression and suicidality are not strictly tethered.

Suicide ideation resolved in our TAU condition, consistent with the notion that ideation is responsive to events or processes that occurred with the passage of time for participants with access to 'usual services'. We do know that at 6 weeks the TAU participants were more likely to make more calls to the helpline service compared with participants in the internet alone condition, but not to the other conditions. Eighty-one per cent of TAU participants reported that they made at least one call, and approximately $55 \%$ had made three or more calls. These calls may have contributed to the suicide resolution in this and the other two conditions. However, these spontaneous calls were unlikely to be responsible for the differences in depression resolution, given that the internet alone group also showed drops in depression levels.

The finding that all groups showed suicide resolution, however, does not rule out the possibility that specific psychological or proactive interventions will support faster resolution of suicide ideation symptoms than the interventions reported here. Indeed, the intervention developed by van Spijker et $a l^{7}$ may be one of these specific interventions.

\section{Limitations of the study}

One explanation of the failure to find an effect of intervention type on suicide ideation outcomes is that these findings are due to the regression to the mean. As all scores were initially high at baseline, they would be expected to revert to the mean on the second occasion of measurement. Intervention completion rates were lower in our study relative to some other trials of webbased treatments for depression ${ }^{26}$ but not of other telephone-based services. ${ }^{3}$ The reasons for this are unclear. The current trial was a true effectiveness trial employing a volunteer workforce for recruitment and call back, and hence might be expected to be associated with greater recruitment and adherence problems relative to the more controlled environment of an efficacy study. ${ }^{27}$ Also, the interventions were short, delivered by non-health professionals and may have constituted the only service received. The measure used to assess suicidality-used items that categorised outcomes dichotomously, and then summed responses as per the scale score recommendations. Moreover, these scores were then further dichotomised in the regression analysis. This is essentially a conservative treatment of data. We did investigate whether other continuous scoring methods might reveal intervention effects. These data did not yield any relationships. The generalisability of the findings to severely suicidal individuals is also limited, given that acutely suicidal or highly distressed callers were specifically not invited to participate. We had relatively low success in recruiting participants to the trial, a finding that reflects the nature of effectiveness trials using targeted samples such as ours ${ }^{27}$ and where a mismatch occurs between the expectation of phoning a crisis centre and being 'diverted' to another program. Finally, we were not able to analyse the influence of other psychological characteristics on treatment outcome, such as hopelessness or support from family, which might affect both the depression and suicidal ideation levels. These measures were not included in our study.

\section{Future research}

Replications of this trial need to be performed to establish consistency of research findings. Furthermore, examination of mediators of change such as hopelessness in larger samples would add significantly to our knowledge of which components of therapy are necessary for reduction of suicide ideation. Given the absence of differences between conditions, it may be that one or more components present in each treatment arm (eg, giving hope, or participating in research) are responsible for change in outcome. Identifying these components and altering their presence by intensifying or omitting them in a new intervention trial would add to our current knowledge of mechanisms of change. Investigation of the causes of reductions in suicide ideation is warranted.

\section{Clinical implications}

Despite the limitations, the current study clearly demonstrated that suicide ideation declined irrespective of intervention, telephone counsellor contact or wait-list. The addition of the TAU control condition is a significant improvement over previous trials. The study demonstrated that, while CBT internet interventions are significantly more effective in resolving depression symptoms than control conditions, these operate no more successfully than current call centre practices in resolving suicide ideation. Suicide ideation reduces significantly more effectively in those individuals who also have resolving depression. The majority of people experiencing suicidal ideation do not receive mental health treatment. ${ }^{28}$ Internet-based or telephone-based interventions have the advantage that they can be easily accessed by large numbers of people, on an anonymous 
basis. In line with Watts $e t a l^{6}$ our findings demonstrate the importance of not excluding suicidal participants from interventions designed to target depression.

Author affiliations

${ }^{1}$ School of Medicine, The Black Dog Institute, University of New South Wales, Sydney, New South Wales, Australia

${ }^{2}$ Centre for Mental Health Research, Australian National University, Canberra, Australian Capital Territory, Australia

${ }^{3}$ Orygen Youth Health, University of Melbourne, Melbourne, Victoria, Australia

Acknowledgements Nicole Burgess coordinated and conducted data collection for the trial. Dawn O'Neil was involved in obtaining funding for the trial and coordinating Lifeline Australia's collaboration with ANU. Associate Professor Richard O'Kearney was a member of LF's PhD supervisory panel. Trevor Carlyon was also involved in coordinating Lifeline staff and volunteer involvement in the trial and supported Nicole in her role. The e-hub team supported the trial implementation. We acknowledge the contributions of the Lifeline counsellors who participated in this trial.

Contributors HC, KMG and AJM conceived the study; LF, HC, KMG and AJM designed the study; HC and TD drafted the initial manuscript; LF, PJB and AJM prepared and cleaned the data and conducted initial data analysis. All authors contributed to the revised drafts.

Funding Australian Research Council Linkage Grant (LP0667970), 2006, 3 years, \$279 325 (Christensen, Griffiths, Mackinnon, Smith), Internet technologies, health informatics, and statistical models, and new delivery platforms in call centres (CIA). HC and PB were supported by NHMRC 525411 and NHMRC Early Career Fellowship 1035262. KMG is supported by NHMRC Fellowship No. 525413.

Competing interests None.

Ethics approval Ethics approval for the trial was granted by the Australian National University Human Research Ethics Committee (Protocol no. 2007/12).

Provenance and peer review Not commissioned; externally peer reviewed.

Data sharing statement No additional data are available.

\section{REFERENCES}

1. Goldberg D, Hillier V. A scaled version of the General Health Questionnaire. Psycholog Med 1979;9:139-45.

2. Lifeline Australia. Lifeline Australia Annual Report 2009-2010. Canberra: Lifeline Australia Ltd, 2010.

3. Rhee WK, Merbaum M, Strujbe MJ, et al. Efficacy of brief telephone psychotherapy with callers to a suicide hotline. Suicide Life Threat Behav 2005;35:317-28.

4. Kalafat J, Gould MS, Harrismunfakh JL, et al. An evaluation of crisis hotline outcomes. Part 1: nonsuicidal crisis callers. Suicide Life Threat Behav 2007;37:322-37.

5. Gould MS, Kalafat J, Harrismunfakh JL, et al. An evaluation of crisis hotline outcomes. Part 2: suicidal callers. Suicide Life Threat Behav 2007;37:338-52.

6. Watts S, Newby JM, Mewton L, et al. A clinical audit of changes in suicide ideas with internet treatment for depression. BMJ Open 2012;2:e001558.

7. van Spijker BA, van Straten A, Kerkhof AJ. The effectiveness of a web-based self-help intervention to reduce suicidal thoughts: a randomized controlled trial. Trials 2010;11:25.
8. Farrer L, Christensen H, Griffiths KM, et al. Internet-based CBT for depression with and without telephone tracking in a national helpline: randomised controlled trial. PLOS ONE 2011;6: e28099.

9. Farrer L, Christensen $\mathrm{H}$, Griffiths KM, et al. Web-based cognitive behavior therapy for depression with and without telephone tracking in a national helpline: secondary outcomes from a randomized controlled trial. J Med Internet Res 2012;14:e68.

10. Nock MK, Hwang I, Samson N, et al. Cross-national analysis of the associations among mental disorders and suicidal behavior: findings from the WHO World Mental Health Surveys. PLoS Med 2009;6: e1000123.

11. Andrews G, Slade T. Interpreting scores on the Kessler Psychological Distress Scale (K10). Aust N Z J Public Health 2001;25:494-7.

12. Christensen $H$, Griffiths $K M$, Jorm AF. Delivering interventions for depression by using the internet: randomised controlled trial. $B M J$ 2004;328:265.

13. Tennant $\mathrm{C}$. The general health questionnaire: a valid index of psychological impairment in Australian populations. Med J Aust 1977;2:392-4.

14. Goldney RD, Smith S, Winfield $\mathrm{AH}$, et al. Suicidal ideation: its enduring nature and associated morbidity. Acta Psychiat Scandin 1991;83:115-20.

15. Goldney RD, Wilson D, Dal Grande E, et al. Suicidal ideation in a random community sample: attributable risk due to depression and psychosocial and traumatic events. Aust N Z J Psychiat 2000;34:98-106.

16. Goldney R, Winefield A, Saebel J, et al. Anger, suicidal ideation, and attempted suicide: a prospective study. Comprehen Psychiat 1997;38:264-8.

17. Goldney RD, Winefield $\mathrm{AH}$, Tiggemann $\mathrm{M}$, et al. Suicidal ideation in a young adult population. Acta Psychiatr Scandin 1989;79: 481-9.

18. Watson D, Goldney R, Fisher L, et al. The measurement of suicidal ideation. Crisis 2001;22:12-14.

19. Radloff LS. The CES-D scale: a self-report depression scale for research in the general population. Appl Psycholog Meas 1977;1:385-401.

20. Roberts RE, Vernon SW. The Center for Epidemiologic Studies Depression Scale: it's use in a community sample. Am J Psychiat 1983;140:41-6.

21. Lovibond SH, Lovibond PF. Manual for the depression anxiety stress scales. Sydney: Psychology Foundation 2nd edn, 1995.

22. Antony MM, Bieling PJ, Cox, et al. Psychometric properties of the 42-item and 21-item versions of the Depression Anxiety Stress Scales (DASS) in clinical groups and a community sample. Psycholog Assess 1998;10:176-81.

23. Lovibond SaLP. The structure of negative emotional states: Comparison of the Depression Anxiety Stress Scales (DASS) with the Beck Depression and Anxiety Inventories. Behav Res Ther 1995;33:8.

24. Thompson EA, Mazza JJ, Herting JR, et al. The mediating roles of anxiety depression, and hopelessness on adolescent suicidal behaviors. Suicide Life Threat Behav 2005;35:14-34.

25. Cuijpers P, de Beurs DP, van Spijker BA, et al. The effects of psychotherapy for adult depression on suicidality and hopelessness: a systematic review and meta-analysis. J Affect Disord 2013;144:183-90

26. Christensen $\mathrm{H}$, Griffiths $\mathrm{KM}$, Farrer $\mathrm{L}$. Adherence in internet interventions for anxiety and depression. J Med Internet Res 2009;11:e13.

27. Flay BR. Efficacy and effectiveness trials (and other phases of research) in the development of health promotion programs. Prev Med 1986;15:451-74.

28. Michelmore L, Hindley P. Help-seeking for suicidal thoughts and self-harm in young people: a systematic review. Suicide Life Threat Behav 2012;42:507-24. 